\title{
130th anniversary of Institut Pasteur: celebrating science
}

\author{
Marie-Lise Gougeon ${ }^{1} \cdot$ Maxime Schwartz ${ }^{2} \cdot$ Thomas Brunner $^{3}$ - David M. Ojcius ${ }^{4}$
}

Received: 10 April 2019 / Accepted: 10 April 2019 / Published online: 25 April 2019

(c) Springer Nature Limited 2019

The Institut Pasteur is an internationally renowned research center that is committed to advancing scientific knowledge at all levels of life, from molecules to individuals and populations, combining multidisciplinary strategies, and relying on an international network of 33 institutes located on five continents. The Institut Pasteur is engaged in responding to the major public health challenges of today, including endemic and emerging infections. Discoveries of the institute improve prevention, diagnosis and treatment of infectious diseases.

One hundred and thirty years ago, the Institut Pasteur opened its doors, following Louis Pasteur's successful international appeal for funds. This special issue celebrates Science conducted at the Institut Pasteur today.

The founding of Institut Pasteur resulted from Louis Pasteur's success in vaccination against rabies. On 1 March 1886, an enthusiastic Academy of Sciences in Paris launched a worldwide fund-raising campaign for the creation of an institute to vaccinate people bitten by rabid animals. This campaign was met with resounding success, and the institute, the Institut Pasteur, was inaugurated on 14 November 1888. Due to the large sums of money collected, Pasteur decided to widen his goals. The institute would not simply be "a center for vaccination against rabies" but rather, as Pasteur wrote in his inaugural address: "Our institute will be a free clinic for rabies treatment, a research center for infectious diseases, and a teaching center". Over the years the Institut Pasteur became one of the most important

Marie-Lise Gougeon

marie-lise.gougeon@pasteur.fr

1 Innate Immunity and Viruses Unit, Infection and Epidemiology Department, Institut Pasteur, Paris 75015, France

2 Institut Pasteur, Paris 75015, France

3 Biochemical Pharmacology, Department of Biology, University of Konstanz, Konstanz, Germany

4 Department of Biomedical Sciences, Arthur A. Dugoni School of Dentistry, University of the Pacific, San Francisco, USA institutions devoted entirely to the life sciences and public health. Among the many discoveries made at the Institut Pasteur, let us recall some that have contributed most to the reputation of the institute.

At Pasteur's time, diphtheria was one of the main causes of childhood mortality. In 1888, the year the institute opened its doors, two of Pasteur's collaborators, Émile Roux and Alexandre Yersin, discovered the first bacterial "poison", diphtheria toxin, secreted by a bacillus. Shortly thereafter, in 1894, in parallel with Emil von Behring in Germany, Roux and his collaborators showed that children with diphtheria could be cured by inoculation of sera from horses previously immunized with the toxin and thus containing what was to be named "antibodies" against the toxin. This was an early example of serotherapy. Several years later, between 1924 and 1926, another scientist at Institut Pasteur, Gaston Ramon, showed that inoculation of inactivated toxin immunized children against diphtheria. He obtained a similar result for tetanus. Inactivated toxins continue to form the basis for modern diphtheria and tetanus vaccines.

Bacillus Calmette-Guérin (BCG) is another well-known vaccine developed at Institut Pasteur. This vaccine was developed by Albert Calmette and Camille Guérin, who attenuated a tuberculosis bacillus by cultivating it on potato slices imbibed with biliary salts for 13 years. BCG, first used in 1921, was to become for a long time the most widely used vaccine in the world. A vaccine against yellow fever was later developed, in 1934, by Jean Laigret at the Institut Pasteur in Dakar, and one against poliomyelitis in 1957 by Pierre Lépine in Paris. More recently, in 1985, the group of Pierre Tiollais developed a vaccine against hepatitis $\mathrm{B}$, the first vaccine produced by genetic engineering.

The field of Immunology emerged to understand the mechanisms whereby vaccines induce immunity. The work of the German scientist Emil von Behring (Nobel prize, 1901) and Emile Roux on "antitoxins" was at the origin of the concept of humoral immunity further developed by Paul Ehrlich in Germany; while Elie Metchnikoff, who studied at the Institut Pasteur the role of phagocytes in the defense 
against pathogenic bacteria, is considered as the father of cellular immunity. Ehrlich and Metchnikoff shared a Nobel prize in 1908; while another scientist of the Institut Pasteur, Jules Bordet, won the prize in 1919 for his work on the mechanisms of action of antibodies and the role of 'complement proteins'.

In his time, Louis Pasteur had strongly contributed to the notion that infectious diseases are caused by microorganisms. Although Robert Koch (Nobel prize, 1905) and his school identified the microbes responsible for most of the major diseases of the time-such as tuberculosis, cholera, diphtheria, and tetanus - the plague bacillus was identified in Hong Kong by Alexandre Yersin. As early as 1880, before joining the Institut Pasteur, Alphonse Laveran (Nobel prize, 1907) had shown that a parasite caused malaria. Closer to our times, the AIDS virus HIV-1 was isolated at the Institut Pasteur in 1983 by the group of Luc Montagnier (Nobel prize, 2008, with Françoise Barré-Sinoussi).

Following Yersin's discovery of the plague bacillus, another Pasteur scientist, Paul-Louis Simond, demonstrated in 1898 the role of fleas in transmitting this bacillus, especially from infected rats. Other Pasteur scientists were involved in elucidating the role of arthropods in the transmission of infectious diseases. Among them was Charles Nicolle (Nobel prize, 1928), who showed in 1909 at the Institut Pasteur of Tunis that typhus is transmitted by body lice.

With due respect to major discoveries made in other countries and, in particular, the elucidation of the DNA structure by James Watson and Francis Crick (Nobel prize, 1962), the Institut Pasteur was at the forefront of laying the foundations for molecular biology. André Lwoff, Jacques Monod and François Jacob-who shared the Nobel prize in 1965-revealed a mechanism for the regulation of gene expression. Their research, which dealt with the adaptation of bacteria to different growth conditions, was seminal in the understanding of a host of other biological processes, such as cell differentiation and carcinogenesis.

This special issue starts with a historical review by JeanMarc Cavaillon and Sandra Legout, which describes how the five musketeers of Louis Pasteur (Duclaux, Chamberland, Roux, Grancher, and Metchnikoff) contributed to the principal discoveries of Pasteur, including development of the first vaccines against avian cholera, anthrax, swine erysipelas and rabies.

Plague is a vector-borne disease caused by Yersinia pestis. It emerged less than 6000 years ago from an enteric bacterial ancestor and is a highly remarkable model for understanding of the evolution of pathogenic bacteria and bacterial strategies to subvert host immunity, as reviewed by Christian Demeure et al.

Uncontrolled bacterial infection leads to pathological conditions, such as sepsis, which remains a contemporary threat. Jean-Marc Cavaillon and Fabrice Chretien remind us that sepsis is an old disease but a new WHO global health priority. They discuss the major contribution of Ignaz Semmelweis, who demonstrated that sepsis is a preventable contagious disease, but note the continuing failure to introduce effective new therapies or better understand the physiopathology of sepsis.

Marco Bellinzoni et al. address the question of how physiological signaling pathways are involved in control of essential cellular processes by a bacteria, such as mycobacteria, and especially the involvement of O-phosphosignaling.

Interestingly, recent annotation of the complete genome sequence of the entire genus Legionella, which comprises over 60 species, revealed that legionellae acquisition of "eukaryotic genes" is an ongoing process that could lead to the emergence of new human pathogens from the environment, as discussed by Carmen Buchrieser et al.

Fungal infections kill more than one million people every year and these neglected infections are very difficult to cure even when validated antifungal treatments are available. The development of new antifungals and diagnostics requires better insights on the biology of fungal pathogens in humans. Guilhem Janbon et al. review the epidemiology and pathophysiology of fungal infections, the role of fungi on human microbiota and immune responses and the genetic basis of increased susceptibility to fungal infections.

Chronic inflammatory diseases cause significant morbidity, and our current understanding of the pathogenic mechanisms leading to disease is limited. Elisabetta Bianchi and Lars Rogge highlight the important contribution of genome-wide association studies (GWAS), which brought to the forefront many genes of the immune system linked to signaling pathways, especially from the IL-23/IL-17 pathway.

Chiara Zurzolo and Jost Enninga stress that only a profound understanding of the structure and function of cells will allow an understanding of what happens in pathological conditions. This can be achieved by combining cellular microbiology and quantitative cell biology approaches, which help to reveal a molecular understanding of cellular events in disease progression.

Finally, Stanislas Pol and Sylvie Lagaye review the amazing history of hepatitis $\mathrm{C}$ virus (HCV) chronic infection, which required 30 years of reciprocal interactions between clinicians and scientists leading to the discovery of the virus, the development of diagnostic tools, predictors of specific immune responses, and finally, effective treatment with oral antivirals that inhibit HCV infection, allowing the cure of all infected subjects.

This collection of articles provides only a glimpse of work carried out at the Institut Pasteur, but shows that the tradition of studying infectious diseases and public health initiated by Louis Pasteur 130 years ago are alive and well at his Institut today. 